The Astrophysical JouRnal, 480:196-202, 1997 May 1

(C) 1997. The American Astronomical Society. All rights reserved. Printed in U.S.A.

\title{
EINSTEIN RADII FROM BINARY-SOURCE LENSING EVENTS
}

\author{
Cheongho Han and Andrew Gould ${ }^{1}$ \\ Department of Astronomy, Ohio State University, 174 West 18th Avenue, Columbus, OH 43210; cheongho@payne.mps.ohio-state.edu, \\ gould@payne.mps.ohio-state.edu \\ Received 1996 April 10; accepted 1996 November 18
}

\begin{abstract}
We show that the Einstein ring radius and transverse speed of a lens projected on the source plane, $\hat{r}_{\mathrm{E}}$ and $\hat{v}$, can be determined from the light curve of a binary-source event, followed by the spectroscopic determination of the orbital elements of the source stars. The determination makes use of the same principle that allows one to measure the Einstein ring radii from finite-source effects. For the case when the orbital period of the source stars is much longer than the Einstein timescale, $P \gg t_{\mathrm{E}}$, there exists a single twofold degeneracy in determining $\hat{r}_{\mathrm{E}}$. However, when $P \lesssim t_{\mathrm{E}}$ the degeneracy can often be broken by making use of the binary-source system's orbital motion. For $\sim 8 \%$ of all lensing events with sources $V<20$ seen toward the Large Magellanic Cloud (LMC), one can unambiguously determine whether the lenses are Galactic or whether they lie in the LMC itself. In addition, we propose to include eclipsing binaries as sources for gravitational lensing experiments.
\end{abstract}

Subject headings: binaries: close — gravitational lensing — relativity

\section{INTRODUCTION}

There are many different effects that make a light curve of a microlensing event deviate from its characteristic achromatic and symmetric form: luminous lenses (Kamionkowski 1995; Buchalter, Kamionkowski, \& Rich 1996), differential amplification during close encounters (Gould 1994; Nemiroff \& Wickramasinghe 1994; Witt \& Mao 1994; Witt 1995; Loeb \& Sasselov 1995; Gould \& Welch 1996), parallax effects caused by the Earth's orbital motion (Gould 1992; Alcock et al. 1995; Buchalter \& Kamionkowski 1997), and finally binary-lens events (Mao et al. 1994; Axelrod et al. 1994; Udalski et al. 1994; Mao \& Di Stefano 1995; Alard, Mao, \& Guibert 1995; Alcock et al. 1997a). Whenever any of these distortions is detected, it provides information about the physical parameters of individual lenses: distance to the lens for luminous lens, lens proper motion, $\mu=v / D_{\mathrm{ol}}$, for differential amplification, observer-plane projected Einstein ring radius, $\tilde{r}_{\mathrm{E}}=$ $\left(D_{\mathrm{os}} / D_{1 \mathrm{~s}}\right) r_{\mathrm{E}}$, for parallax, and the geometry of a lens binary system, and sometimes the proper motion for binary lens events. Here $v$ is the speed of the lens relative to the Earthsource line of sight, and the physical and angular Einstein ring radius are related to the physical parameters of the lens by

$$
r_{\mathrm{E}}=\left(\frac{4 G M_{L}}{c^{2}} \frac{D_{\mathrm{ol}} D_{1 \mathrm{~s}}}{D_{\mathrm{os}}}\right)^{1 / 2}, \quad \theta_{\mathrm{E}}=\frac{r_{\mathrm{E}}}{D_{\mathrm{ol}}},
$$

where $D_{\mathrm{ol}}, D_{\mathrm{ls}}, D_{\mathrm{os}}$ are the distances between the observer, source, and lens, $r_{\mathrm{E}}$ is the physical size of the Einstein ring, and $M_{L}$ is the mass of the lens.

The light curve can be also distorted when the source is composed of a binary system, resulting in a binary-source event (Griest \& Hu 1992). A wide variety of binary-source events is possible, ranging from those where the companion is not amplified and the event is therefore a simple blend to those where the binary induces extreme fluctuations in the

\footnotetext{
${ }^{1}$ Alfred P. Sloan Foundation Fellow.
}

light curve. The factors that affect the light-curve shapes of binary-source events include the angular size of the projected separation between the source stars, source trajectories within the Einstein ring, the angular size of the Einstein ring projected onto the source plane, and the orbital motion. Griest \& Hu (1992) concentrated on binary-source events for which the binary period is long compared to the event timescale. They briefly discussed the possibility of events with short-period binary sources and illustrated the dramatic oscillation which these could in principle generate. They noted, however, that for the expected lens parameters the amplitude of these oscillations would be extremely small.

In the present paper, by contrast, we concentrate on binary-source events where the binary stars move substantially, i.e., short-period binaries. We show that the Einstein ring radius and the transverse speed projected on the source plane, $\hat{r}_{\mathrm{E}}$ (source-plane Einstein ring radius) and $\hat{v}$ (sourceplane transverse speed), can be determined from the light curve of a short-period binary-source event, provided that the observations are followed by spectroscopic determination of the binary-source orbital elements. Throughout this paper, we use a caret $\left(^{\wedge}\right)$ to represent a quantity projected onto the source plane. The source-plane Einstein radius and transverse speed are defined by

$$
\hat{r}_{\mathrm{E}}=D_{\mathrm{os}} \theta_{\mathrm{E}}=r_{\mathrm{E}} \frac{D_{\mathrm{os}}}{D_{\mathrm{ol}}}, \quad \hat{v}=\frac{\hat{r}_{\mathrm{E}}}{t_{\mathrm{E}}},
$$

where $t_{\mathrm{E}}=r_{\mathrm{E}} / v$ is the Einstein ring crossing time. Note that when the source distance is known (e.g., for observations toward the Large Magellanic Cloud [LMC]), measuring $\hat{r}_{\mathrm{E}}$ and $\hat{v}$ is equivalent to measuring $\theta_{\mathrm{E}}$ and the proper motion $\mu$ since $\theta_{\mathrm{E}}=\hat{r}_{\mathrm{E}} / D_{\mathrm{os}}$ and $\mu=\hat{v} / D_{\mathrm{os}}$. The basic principle that makes this measurement possible is that a binary acts like an enormous finite source and therefore is much more susceptible to finite-source effects than is a single star. Once the proper motion is measured, one can clearly separate Galactic versus LMC self-lensing events because of the large difference in the proper motions between the two populations of events (see $\S 6$ ). 
Short-period binary sources are important for several reasons. First, it is these events that allow one to unambiguously measure the proper motion. For long-period binaries, one can determine $\hat{r}_{\mathrm{E}}$, but with a twofold degeneracy (see $\S 3$ ). Second, for lenses in the LMC the amplitude of the oscillations in the flux due to the binary-source effect is expected to be of order $10 \%$ and would be easily observable (see $\S 5$ ). Within the framework of the standard model, LMC events are expected to be relatively rare. However, Sahu (1994) has argued that essentially all the events currently detected toward the LMC are due to LMC lenses. This may seem unlikely in view of the large optical depth (Alcock et al. 1996a), but it is nevertheless important to test this hypothesis since the alternative is that the halo is composed in large part of massive compact halo objects (MACHOs). As we show in $\S 5$, about $10 \%$ of LMC sources are short-period binaries. These binaries allow a direct test of the Sahu (1994) hypothesis. Third, while the effects are smaller for Galactic lenses, they are not negligible. The significant advances now being made in rapid detection and follow-up observations (Pratt et al. 1996a; Albrow et al. 1996; Alcock et al. 1996b) open the possibility that even 1\% or $2 \%$ oscillations caused by binary sources may soon be measurable. Finally, binary sources are competitive with other methods of measuring proper motions toward the Galactic bulge.

\section{PROPER MOTION FROM A BINARY-SOURCE EVENT}

The light curve of a binary-source event is the sum of light curves of individual sources and is represented by

$$
F=\sum_{j=1}^{2} A_{j} F_{0, j}, \quad A_{j}=\frac{u_{j}^{2}+2}{u_{j}\left(u_{j}^{2}+4\right)^{1 / 2}},
$$

where $j=1,2$ denote the primary and secondary source stars, $F_{0, j}$ are the unamplified fluxes, and $u_{j}$ are the projected locations of the source stars with respect to the lens in units of $r_{\mathrm{E}}$.

When stars in a binary have negligible orbital motion (long-period binary), the source stars move along a straight line due to the source-lens-observer transverse motion. Then the source positions are well approximated by

$$
u_{j}^{2}=\omega^{2}\left(t-t_{0, j}\right)^{2}+\beta_{j}^{2},
$$

where $\omega=t_{\mathrm{E}}^{-1}, \beta_{j}$, and $t_{0, j}$ are the impact parameters and the times of maximum amplification for individual source stars. Note that the Einstein timescale is same for both sources. Then the projected (two-dimensional) separation between two stars is related to $\hat{r}_{\mathrm{E}}$ by

$$
\ell=\ell_{1}+\ell_{2}=\hat{r}_{\mathrm{E} \pm}\left[\left(\omega \Delta t_{0}\right)^{2}+\Delta \beta_{ \pm}^{2}\right]^{1 / 2},
$$

where $\ell_{1}=\ell /\left(\mathscr{Q}_{M}+1\right)$ and $\ell_{2}=\mathscr{Q}_{M} \ell_{1}$ are the separations between the center of mass $(\mathrm{CM})$ and the individual sources. Here $\mathscr{Q}_{M}=M_{1} / M_{2}$ is the mass ratio between the source stars. If the individual source star light curves can be decomposed from the obtained light curve, one can measure the values of $\Delta t=\left|t_{0,1}-t_{0,2}\right|$ and $\Delta \beta_{ \pm}=\left|\beta_{1} \pm \beta_{2}\right|$. In addition, the separation $\ell$ can be measured from the followup spectroscopy (see below). Therefore, one can determine $\hat{r}_{\mathrm{E}}$ from the relation in equation (2.3), but with a twofold degeneracy. In $\S 3$, we discuss the twofold degeneracy in $\hat{r}_{\mathrm{E}}$ induced by the ambiguity in the impact parameter difference, $\Delta \beta_{ \pm}$.
On the other hand, when the orbital motion of a binary is important (short-period binary), the positions of the source stars are the combination of the linear transverse motion of the $\mathrm{CM}$ and the orbital motion of component stars around the CM:

$$
u_{j}^{2}=u_{\mathrm{CM}}^{2}-2 u_{\mathrm{CM}}\left(\frac{\ell_{j}}{\hat{r}_{\mathrm{E}}}\right) \cos \theta_{j}+\left(\frac{\ell_{j}}{\hat{r}_{\mathrm{E}}}\right)^{2},
$$

where $\theta_{j}$ is the lens-CM-source angle. The values of $u_{j}$ and $u_{\mathrm{CM}}$ are determined from the light curve. Then, once the orbital motion, i.e., $\ell_{j}\left[\theta_{j}(t)\right]$, is known, one can determine $\hat{r}_{\mathrm{E}}$.

The projected separation between source stars is related to the intrinsic (three-dimensional) separation $\ell_{0}$ by

$$
\ell=\ell_{0}\left|\frac{\sin \left(\Theta+\psi_{P}\right)}{\sin \Omega}\right| \cos i,
$$

where $\Theta$ is the true anomaly, $\Omega$ is the position angle to a star measured from the ascending node, $i$ is the inclination angle, and $\psi_{p}$ is the longitude of the periastron measured from the ascending node. Here the position angle is measured along the projected orbital plane, while the longitude is measured along the true orbital plane. The intrinsic separation between the stars is given by

$$
\ell_{0}(\Theta)=\frac{a\left(1-\epsilon^{2}\right)}{1+\epsilon \cos \Theta} .
$$

Here $\epsilon=\left(1-b^{2} / a^{2}\right)^{1 / 2}$ is the eccentricity, where $a$ and $b$ are the semimajor and semiminor axes of the orbit. When the orbit is seen face-on $\left(i=0^{\circ}\right), \Omega=\Theta+\psi_{p}$, and thus $\ell=\ell_{0}$. For a gravitationally lensed binary-source system, the individual source-star masses can be estimated to a first approximation from the luminosities and colors. One can then further constrain the source-star masses by determining the stellar types from follow-up spectroscopy. To determine the luminosities and colors, the individual light curves must be extracted from the observed light curve or must be inferred from follow-up spectroscopy. With the known individual masses, the orbital period, and the semimajor axis, the orbital elements, e.g., $\psi_{p}, i, \epsilon$, and $\Omega$, can be determined from the radial velocity curve, which can be constructed from follow-up spectroscopy.

For the determination of the orbital elements, a single spectral line from either star of the binary will be enough to constrain the stellar motion provided that the mass ratio $M_{2} / M_{1}$ is known (Smart 1962). For example, the maximum and minimum values of radial velocities for a circular orbit are

$$
\left\{\begin{array}{l}
v_{r, \max }=\frac{2 \pi a_{1} \sin i}{P}+v_{0}, \\
v_{r, \min }=-\frac{2 \pi a_{1} \sin i}{P}+v_{0},
\end{array}\right.
$$

where $v_{0}$ is the radial velocity of the center of mass. Then the inclination angle is determined by $\sin i=\left(P / 4 \pi a_{1}\right)$ $\left(v_{r, \max }-v_{r, \min }\right)$, where $P, v_{r, \max }$, and $v_{r, \min }$ are measured from the radial velocity curve and $a_{1}$ is known from $P, M_{1}$, $M_{2}$, and Kepler's third law: $a_{1}^{3}=P^{2} M_{2}^{3} /\left(M_{1}+M_{2}\right)^{2}$. If the orbit is very close to face-on, then $v_{r, \text { max }} \sim v_{r, \min } \sim 0$, and thus it would be difficult to confirm the binary nature of the 
source star. However, this case is geometrically rare. Once all these orbital parameters are known, one can find the projected separation $\ell$ from equations (2.5) and (2.6).

\section{DEGENERACY}

There exists a degeneracy in the determination of $\hat{r}_{\mathrm{E}}$ for long-period binary source events. In general, there are two types of degeneracies in the binary-source lens geometry. The first type occurs because the direction of source motion (with respect to the lens) is not known. Fortunately, this type of degeneracy does not affect the the determination of $\hat{r}_{\mathrm{E}}$ because of the radial symmetry of Einstein rings. The other type of degeneracy, which does affect the determination of $\hat{r}_{\mathrm{E}}$, arises because the component of the separation normal to the source motion can have two possible values depending on whether the sources are located on the opposite $\left(\Delta \beta_{+}=\beta_{1}+\beta_{2}\right)$ or at the same $\left(\Delta \beta_{-}=\left|\beta_{1}-\beta_{2}\right|\right)$ side with respect to the lens, resulting in two possible values of source-plane Einstein ring size, $\hat{r}_{\mathrm{E}+}$ and $\hat{r}_{\mathrm{E}-}$. An illustration of two degenerate lens-source positions (small filled circles) is shown in Figures $1 a$ and $1 b$.

However, the degeneracy can be broken for binaries with short periods, i.e., $P \lesssim 10 t_{\mathrm{E}}$. The principle is simple: when the orbital motion is important, the light curves resulting from the two degenerate lens geometries will be different. In Figures $1 a$ and $1 b$, we present tracks of the actual orbiting source stars (dotted lines) compared to the hypothetical straight-line tracks assuming no orbital motion (solid lines). The resulting light curves taking the orbital motions into consideration are shown in Figures $1 c$ and $1 d$ (dotted curves), and they are compared to those without orbital motions (solid curves). In the example, we assume that the event has $t_{\mathrm{E}}=20$ days and the binary is observed to have $F_{0,1}=F_{0,2}, P=100$ days, and inclination angle $i=0^{\circ}$.
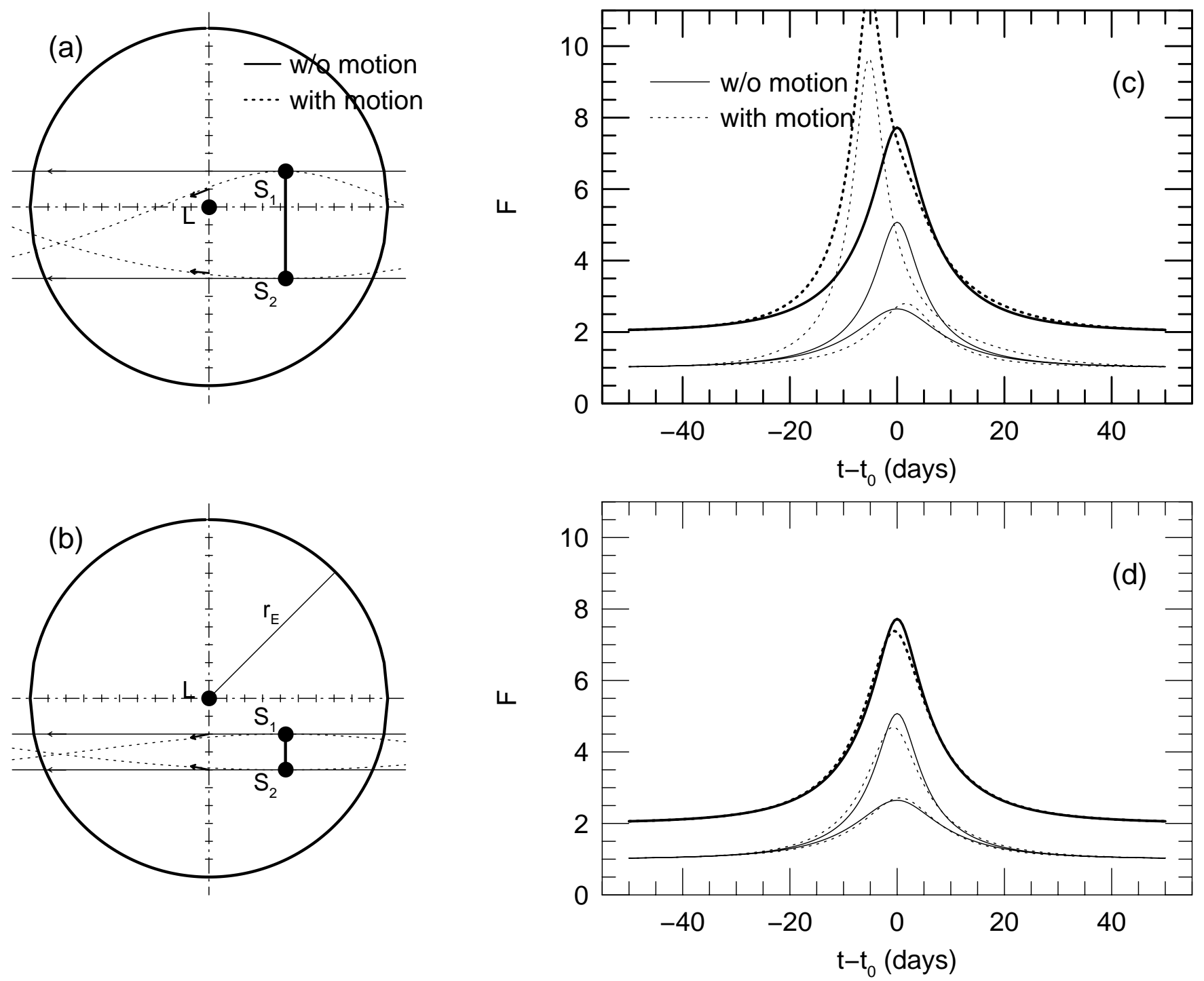

FIG. 1.-Breaking the degeneracy in $\hat{r}_{\mathrm{E}}$. Without orbital motion, the lens geometries $(a)$ and $(b)$ would produce the same light curves. However, when the orbital motion is important, the light curves resulting from the two degenerate lens geometries will be different as shown in the $(c)$ and $(d)$. Light curves with and without orbital motion are marked by dotted and solid lines, respectively. Light curves of the primary and secondary stars are presented by thin lines, while the sum of fluxes from both the primary and secondary is shown with thick lines. The tracks of the actual orbiting source stars (dotted lines) are compared to the straight-line tracks (solid lines) assuming no motion. In the example event, we adopt $t_{\mathrm{E}}=20$ days and $P=100$ days with a face-on circular orbit. 
For simplicity, we adopt a circular orbit with a mass ratio $\mathscr{Q}_{M}=1$, i.e., the radii of orbital motion $a_{1}=a_{2}=a / 2$; where $a=0.8 \mathrm{AU}$ is the semimajor axis, and the separation of $\ell_{j}=a_{j}=$ constant, corresponding to source mass sum of $M=M_{1}+M_{2}=a^{3} / P^{2}=6.82 M_{\odot}$. The projected separation between the two source stars in units of $\theta_{\mathrm{E}}$ is $\hat{\lambda}=0.6$ and 0.2 for the cases when source stars are located at the same and opposite sides with respect to the lens, as shown in Figures $1 a$ and $1 b$. The circle around the lens represents the Einstein ring. Then there are two possible values of $\hat{r}_{\mathrm{E}}=a / \hat{\lambda}: \hat{r}_{\mathrm{E}+}=1.3 \mathrm{AU}$ and $\hat{r}_{\mathrm{E}-}=4.0 \mathrm{AU}$. The degeneracy is clearly broken since the light curve (Fig. 1c) is radically different from Figure 1d.

\section{SHORT-PERIOD BINARY EVENT LIGHT CURVES}

We have shown that short-period binaries are important in determining the proper motion. In this section, we derive an analytic form of the short-period binary-source event light curve to better understand this type of events. If the angular separation between the binary-source stars is small compared to the source-lens separation, one can treat the difference between the center of light (CL) and the position of each source star as a perturbation $\delta u_{j}$ of the case when both stars are at the CL, i.e.,

$$
\begin{aligned}
u_{j} & =\left(u^{2}-2 u \hat{\lambda}_{j} \cos \theta_{j}+\hat{\lambda}_{j}^{2}\right)^{1 / 2} \sim u+\delta u_{j}, \\
\delta u_{j} & =-\hat{\lambda}_{j} \cos \theta_{j}+\frac{1}{2 u_{j}} \hat{\lambda}_{j}^{2} \sin ^{2} \theta_{j},
\end{aligned}
$$

where $u=u_{\mathrm{CL}}$ and $u_{j}$ are the locations of the CL and of each source star in units of $r_{\mathrm{E}}$, and $\theta_{j}$ is the angle between the lines connecting the $\mathrm{CL}$ with the lens and the $\mathrm{CL}$ with each source (see Fig. 2). Here $\hat{\lambda}_{j}$ are the offsets of the two sources from the CL, i.e., $\hat{\lambda}=\hat{\lambda}_{1}+\hat{\lambda}_{2}$ and $\hat{\lambda}_{1} F_{0,1}=\hat{\lambda}_{2} F_{0,2}$, and

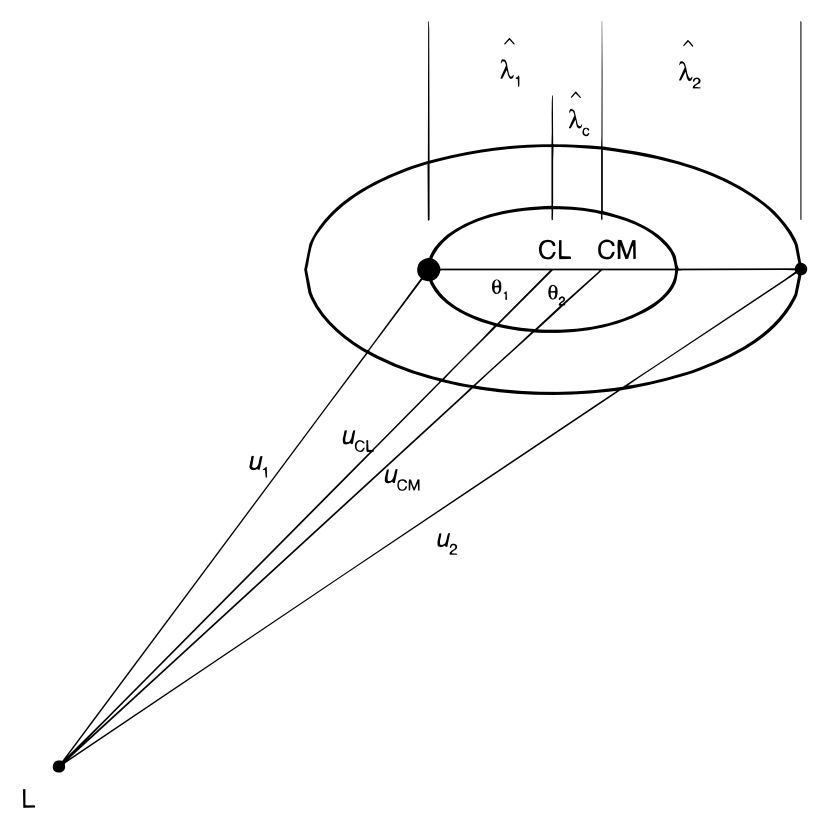

FIG. 2.-Short-period binary-source geometry. Since $u \gg \hat{\lambda}_{j}$, the separations $\delta u_{j} \sim\left|\boldsymbol{u}_{\mathrm{CL}}-\boldsymbol{u}_{j}\right|$ can be treated as a perturbation of the case when both stars are at the center of light (CL). The source stars are denoted by filled circles (bigger for the primary) and the lens is marked by " $L$." Similarly, one can treat the separation $\delta u_{c} \sim\left|\boldsymbol{u}_{\mathrm{CM}}-\boldsymbol{u}_{\mathrm{CL}}\right|$ as a perturbation to take account of the motion of the CL around the center of mass (CM). they are related to the projected physical separations, $\ell_{j}$, by $\hat{\lambda}_{j}=\left(\ell_{j} / r_{\mathrm{E}}\right)\left(D_{\mathrm{ol}} / D_{\mathrm{os}}\right)=\ell_{\mathrm{j}} / \hat{r}_{\mathrm{E}}$. The angles are related by $\theta_{2}=$ $\pi-\theta_{1}$. The corresponding flux perturbation is then

$$
\delta F_{\mathrm{sep}} \sim A^{\prime} \sum_{j=1}^{2} F_{0, j} \delta u_{j}+\frac{A^{\prime \prime}}{2} \sum_{j=1}^{2} F_{0, j} \delta u_{j}^{2},
$$

where

$$
\begin{aligned}
& A^{\prime}=\frac{d A}{d u}=-\frac{8}{u^{2}\left(u^{2}+4\right)^{1 / 2}}, \\
& A^{\prime \prime}=\frac{d^{2} A}{d u^{2}}=-\frac{5 u^{2}+8}{u\left(u^{2}+4\right)} A^{\prime} .
\end{aligned}
$$

By combining equations (4.1) and (4.2), and keeping terms up to second order in $\hat{\lambda}_{j}$, one finds the perturbation term to be

$$
\begin{aligned}
\delta F_{\text {sep }}= & \frac{A^{\prime}}{2 u}\left(F_{0,1} \hat{\lambda}_{1}^{2}+F_{0,2} \hat{\lambda}_{2}^{2}\right) \\
& \times\left[\sin ^{2} \theta_{1}-\frac{5 u^{2}+8}{\left(u^{2}+4\right)} \cos ^{2} \theta_{1}\right] .
\end{aligned}
$$

In the regime where $u \ll 1$, equation (4.4) is approximated by

$$
\delta F_{\text {sep }} \sim \frac{A^{\prime}}{2 u}\left(F_{0,1} \hat{\lambda}_{1}^{2}+F_{0,2} \hat{\lambda}_{2}^{2}\right)\left(3 \sin ^{2} \theta_{1}-2\right),
$$

resulting in the fractional perturbation of

$$
\frac{\delta F_{\text {sep }}}{F} \sim-\frac{1}{2} \frac{3 \sin ^{2} \theta_{1}-2}{\mathscr{Q}_{L}+2+2_{L}^{-1}}\left(\frac{\hat{\lambda}}{u}\right)^{2}
$$

since $A \sim 1 / u$ and $A^{\prime} \sim-1 / u^{2}$ in this regime.

Here $\mathscr{Q}_{L}=F_{0,1} / F_{0,2}$ is the luminosity ratio between two stars and $F=F_{0,1}+F_{0,2}$ is the total flux. This perturbation alone describes the deviation from the single source light curve well enough when the binary pair is composed of stars of similar type so that the location of the CL is close to the the center of mass $(\mathrm{CM})$.

However, the stars are orbiting around the CM not around the CL. Therefore, another perturbation term $\delta F_{c}$ arises due to the difference between the positions between the CM and CL, $\delta u_{c}$. With similar geometry (see Fig. 2), the location of the CM is

$$
\begin{aligned}
& u_{\mathrm{CM}}=\left(u_{\mathrm{CL}}^{2}+2 u_{\mathrm{CL}} \hat{\lambda}_{c} \cos \theta_{1}+\hat{\lambda}_{c}^{2}\right)^{1 / 2} \sim u_{\mathrm{CL}}+\delta u_{c}, \\
& \delta u_{c}=\hat{\lambda}_{c} \cos \theta_{1}+\frac{1}{2 u_{\mathrm{CL}}} \hat{\lambda}_{c}^{2} \sin ^{2} \theta_{1},
\end{aligned}
$$

where the offset between the CL and the CM is

$$
\hat{\lambda}_{c}=\frac{\mathscr{Q}_{L}-\mathscr{Q}_{M}}{\left(1+\mathscr{Q}_{M}\right)\left(1+\mathscr{Q}_{L}\right)} \hat{\lambda} .
$$

Note that for the geometry shown in Figure $2, \mathscr{2}_{L}>\mathscr{2}_{M}$, so $\hat{\lambda}_{c}>0$. The flux difference due to the difference in the positions of the CL and the CM is then approximated by

$$
\begin{aligned}
\delta F_{c}= & \left(F_{0,1}+F_{0,2}\right)\left[A^{\prime} \delta u_{c}+\frac{A^{\prime \prime}}{2} \delta u_{c}^{2}\right] \\
& \sim\left(F_{0,1}+F_{0,2}\right)\left[A^{\prime} \cos \theta_{1} \hat{\lambda}_{c}\right. \\
& \left.+\frac{1}{2 u}\left(A^{\prime} \sin ^{2} \theta_{1}+A^{\prime \prime} u \cos ^{2} \theta_{1}\right) \hat{\lambda}_{c}^{2}\right],
\end{aligned}
$$


by keeping terms up to second order in $\hat{\lambda}_{c}$. For $\hat{\lambda}_{c} \ll u$, the first term always dominates, implying a perturbation,

$$
\frac{\delta F_{c}}{F} \sim-\frac{A^{\prime} \cos \theta_{1}}{A} \hat{\lambda}_{c} .
$$

One then arrives at the final form of the approximation to the total flux,

$$
F=A\left(F_{0,1}+F_{0,2}\right)+\delta F ; \delta F=\delta F_{c}+\delta F_{\text {sep }} .
$$

The leading first terms of the fractional deviation from a single-source event are given by

$$
\frac{\delta F}{F} \sim \frac{\left(\mathscr{Q}_{L}-\mathscr{Q}_{M}\right) \cos \theta_{1}}{\left(1+\mathscr{Q}_{M}\right)\left(1+\mathscr{Q}_{L}\right)}\left(\frac{\hat{\lambda}}{u}\right)-\frac{1}{2} \frac{3 \sin ^{2} \theta_{1}-2}{\mathscr{Q}_{L}+2+\mathscr{2}_{L}^{-1}}\left(\frac{\hat{\lambda}}{u}\right)^{2} .
$$

In Figure 3, we present three examples of short-period

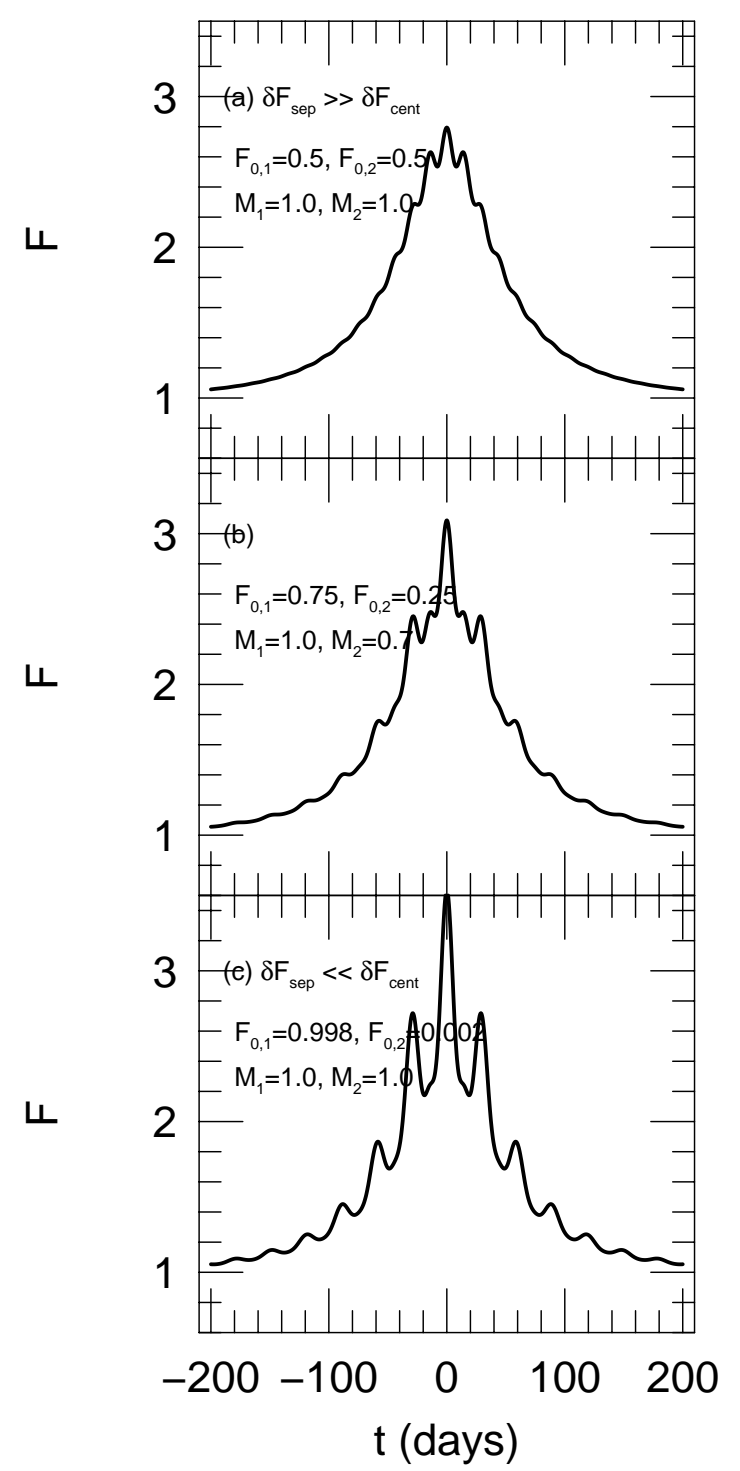

binary-source events: $(a)$ when $\delta F_{\text {sep }} \gg \delta F_{c}$ (e.g., pair of stars with same mass and luminosity), (b) when both perturbations take place, and $(c)$ when $\delta F_{\text {sep }} \ll \delta F_{c}$ (e.g., same mass but $F_{0,1} \gg F_{0,2}$ such as a giant-white dwarf pair). The values of $\delta F / F$ for individual cases are shown in the panels on the right-hand side. All three example events have $\beta=0.4, t_{\mathrm{E}}=100$ days, and orbital period of $P=30$ days, and the orbit is face-on and circular, for simplicity. The masses and luminosities of the individual stars for each case are marked in each panel. The peaks of the curve occur with a frequency of $\sim P / 2$ for $(a)$ events since the varying part of $\delta F_{\text {sep }}$ is proportional to $\sin ^{2} \theta_{1}$, while the frequency is $\sim P$ for $(c)$ events because $\delta F_{c} \propto \cos \theta_{1}$. For the general case in which both perturbations play roles, the perturbation $\delta F_{c} / F \propto u^{-1}$ decays slowly compared to $\delta F_{\text {sep }} / F \propto u^{-2}$, and thus the $\delta F_{c}$ perturbation (period $P$ ) dominates the wings of the light curve while the $\delta F_{\text {sep }}$ perturbation (period $P / 2$ ) dominates near the peak.

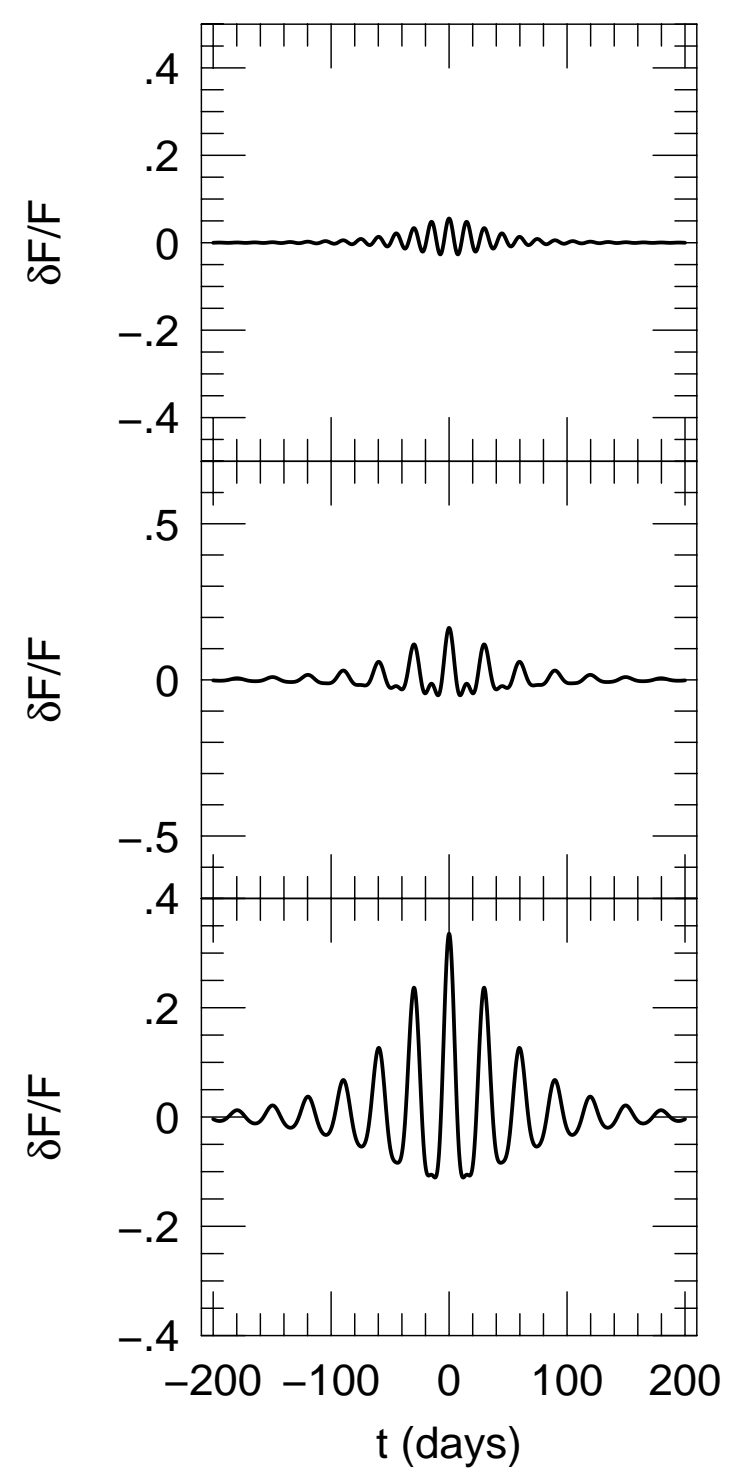

FIG. 3.-Short-period binary-source event light curves: $(a)$ when $\delta F_{\text {sep }} \gg \delta F_{c}$ (e.g., pair of stars with same mass and luminosity), (b) when both perturbations take place, and $(c)$ when $\delta F_{\text {sep }} \ll \delta F_{c}$ (e.g., same mass but $F_{0,1} \gg F_{0,2}$ such as a giant-white dwarf pair). The values of $\delta F / F$ for individual cases are shown at the right side panels. All three example events have $\beta=0.4, t_{\mathrm{E}}=100$ days, and orbital period of $P=30$ days. The mass (in $M_{\odot}$ ) and luminosity of individual stars for each case are marked in each panel. The peaks of the curve occur with a frequency of $P / 2$ for $(a)$ events $\operatorname{since} \delta F_{\text {sep }} \propto \sin ^{2} \theta_{1}$, while the frequency is $P$ for $(c)$ events because $\delta F_{c} \propto \cos \theta_{1}$. For the general case in which both perturbations play roles, the perturbation $\delta F_{c} / F \propto u^{-1}$ decays slowly compared to $\delta F_{\text {sep }} / F \propto u^{-2}$, and thus the $\delta F_{c}$ perturbation (period $P$ ) dominates the wings of the light curve. 


\section{FRACTION OF EVENTS WITH MEASURABLE PROPER MOTION}

What fraction of events have binary sources with periods sufficiently short to give rise to short-period binary-source events, and how large are the oscillations that they generate? The answer to the first question depends only on the source population, while the answer to the second depends primarily on the positions of the lenses. Here, we present rough estimates for these values.

For the LMC, about $\frac{1}{3}$ of the sources with $V<19$ are A stars and $\frac{2}{3}$ are red giants (C. Alcock 1993, private communication). The typical timescale of observed events is $t_{\mathrm{E}} \sim 42$ days (Alcock et al. 1997b; Pratt et al. 1996b). From Tables 2 and 3 of Griest \& Hu (1992) about $18 \%$ of A stars have companions in the period range $1 \lesssim P \lesssim 100$ days and for about $\frac{2}{3}$ of these (or $12 \%$ of all A-star sources), the mass ratio is $1 / \mathscr{Q}_{M}>0.35$. For simplicity we adopt average parameters $P=10$ days, $M=5 M_{\odot}, 1 / \mathscr{Q}_{M}=0.5$, and $\mathscr{Q}_{L} \gg$ 1. Then the leading factor in equation (4.12) $\left(\mathscr{Q}_{L}-\mathscr{Q}_{M}\right) /$ $\left[\left(1+\mathscr{Q}_{M}\right)\left(\mathscr{Q}_{L}\right)\right] \sim \frac{1}{3}$ since $\mathscr{Q}_{L}-\mathscr{Q}_{M} \rightarrow \mathscr{Q}_{L}$ and $1+\mathscr{Q}_{L} \rightarrow \mathscr{Q}_{L}$. By Kepler's third law, the typical separation is $\ell=0.16 \mathrm{AU}$. Then one finds a typical amplitude of fluctuation

$$
\frac{\delta F}{F} \sim \frac{\ell}{3 u \hat{r}_{\mathrm{E}}} \sim \frac{0.1 \mathrm{AU}}{\hat{r}_{\mathrm{E}}},
$$

where we have adopted $u \sim 0.5$ as an average value.

For LMC self-lensing events with a projected speed $v \sim 50 \mathrm{kms}^{-1}$, we find $\hat{r}_{\mathrm{E}}=\left(D_{\mathrm{os}} / D_{\mathrm{ol}}\right) v t_{\mathrm{E}} \sim 1$ AU. Hence, the oscillation is of order $10 \%$. Another $30 \%$ of A stars have companions in the range $0.2<1 / \mathscr{Q}_{M}<0.35$ and so give rise to effects about half this big. We repeat this calculation for red giants using the binary statistics of their F-star progenitors, but restrict attention to periods $10<P<100$ days since closer companions will be destroyed. We find that $\sim 5 \%$ of red giants also give rise to oscillations of $\sim 10 \%$. Thus, $8 \%$ of all source stars should be binaries with periods short enough and companions heavy enough to give rise to effects of order $10 \%$. However, we note that the sources should be brighter than $V \sim 20$ so that the follow-up spectroscopy can be carried out.

For Galactic halo events, the expected source plane Einstein ring radius is $\hat{r}_{\mathrm{E}} \sim 5 r_{\mathrm{E}} \sim 12 \mathrm{AU}$, where we assume that $v=200 \mathrm{kms}^{-1}$ and $D_{\mathrm{ol}}=10 \mathrm{kpc}$. Then the expected fluctuation is $\delta F / F \sim 1.0 \%$, which would be difficult to detect, although perhaps not impossible. However, we stress that for the $8 \%$ of source stars that are short-period binaries even nondetection of the oscillations would be important because it would establish that the lens was in the Galaxy and not in the LMC.

Toward the Galactic bulge field, the typical timescale is shorter, $\sim 20$ days (Alcock et al. 1997a). For bulge selflensing events with $v \sim 200 \mathrm{kms}^{-1}$, a typical source plane
Einstein ring radius is $\hat{r}_{\mathrm{E}} \sim 3$ AU. For periods of $P \sim 20$ days and source mass $M \sim 1.5 M_{\odot}$, one finds a separation of $\ell \sim 0.17$ AU. Typical effects are then $\delta F / F \sim(2 / 3)(0.17 /$ $3)=4 \%$ from equation (5.1), and thus they are easily measurable for giants, among which $\sim 5 \%$ of them have favorable orbital periods (i.e., $10 \leq P \leq 100$ days). However, the absolute number of detectable binaries would be larger compared to that of LMC fields because the spectroscopy could be carried out additionally for some bright turnoff stars. Therefore, we suspect that there may exist several binary-source events in the data set of the current lensing experiments.

Eclipsing binaries provide excellent opportunities to determine $\hat{r}_{\mathrm{E}}$, although the expected event rate is very low. This is because the pure lensing light curve can be easily recovered by dividing the observed light curve by the preevent eclipsing light curve. In addition, the orbital elements of eclipsing binaries can be easily determined because of the known $i=90^{\circ}$. The eclipse light curve can also be used to help constrain the luminosities of the binary sources. Current experiments have identified many eclipsing stars $\left[\sim \mathcal{O}\left(10^{4}\right)\right]$ toward the Galactic bulge and LMC (Grison et al. 1995; Cook et al. 1995; Kałużny et al. 1995), but, unfortunately, they are excluded as gravitational lensing source stars due to their variability. Because of the extra information which is automatically available in the case of eclipsing binaries, they should be reincluded in the lensing search. Luckily enough, there already has been reported a candidate event (EROS2; Ansari et al. 1995) with a microlensingtype light curve superimposed on top of periodic eclipsing binary variability.

\section{IDENTIFICATION OF LENS POPULATION}

Once the values of $\hat{r}_{\mathrm{E}}$ and thus $\hat{v}$ are known, one can strongly constrain the nature of individual lenses. First, for the known distance to source stars, e.g., $d_{\text {bulge }}=8 \mathrm{kpc}$ and $d_{\mathrm{LMC}}=50 \mathrm{kpc}$ toward the Galactic bulge and LMC, respectively, determining $\hat{r}_{\mathrm{E}}$ is equivalent to measuring the proper motion $\mu=\theta_{\mathrm{E}} / t_{\mathrm{E}}$. While the timescale is a function of three parameters, $t_{\mathrm{E}}=t_{\mathrm{E}}\left(M_{L}, v, D_{\mathrm{ol}}\right)$, the Einstein ring is a function of only two, $\theta_{\mathrm{E}}=\theta_{\mathrm{E}}\left(M_{L}, D_{\mathrm{ol}}\right)$, and hence provides less degenerate information. Secondly, once $\mu$ is measured, one can easily distinguish Galactic halo from LMC self-lensing events from the difference in $\mu$ because $\mu_{\text {LMC }} \ll \mu_{\text {halo }}$. In Table 1, we present the expected typical values of $\left\langle\hat{r}_{\mathrm{E}}\right\rangle$ and $\langle\hat{v}\rangle$ for the LMC disk, LMC halo, and Galactic halo events. Also presented are the typical parameters for the geometry, $\left\langle D_{\mathrm{ol}} / D_{\mathrm{os}}\right\rangle$, the lens mass $\left\langle M_{L}\right\rangle$, and the transverse speed, $\langle v\rangle$, which are used in determining the expected $\left\langle\hat{r}_{\mathrm{E}}\right\rangle$ and $\langle v\rangle$. We adopt heavier masses for LMC disk lenses because the lenses are expected to be objects above hydrogenburning limit. As a tool to identify the lens population, $\hat{v}$

TABLE 1

Characteristic Values of $\hat{r}_{\mathrm{E}}$ and $\hat{v}$ For Different Populations of Lenses

\begin{tabular}{cccccccc}
\hline \hline Population & $\left\langle D_{\mathrm{o} 1} / D_{\text {os }}\right\rangle$ & $\begin{array}{c}\left\langle M_{L}\right\rangle \\
\left(M_{\odot}\right)\end{array}$ & $\begin{array}{c}\left\langle r_{\mathrm{E}}\right\rangle \\
(\mathrm{AU})\end{array}$ & $\begin{array}{c}\left\langle\hat{r}_{\mathrm{E}}\right\rangle \\
(\mathrm{AU})\end{array}$ & $\begin{array}{c}\langle v\rangle \\
\left(\mathrm{km} \mathrm{s}^{-1}\right)\end{array}$ & $\begin{array}{c}\left\langle t_{\mathrm{E}}\right\rangle \\
(\mathrm{days})\end{array}$ & $\begin{array}{r}\langle\hat{v}\rangle \\
\left(\mathrm{km} \mathrm{s}^{-1}\right)\end{array}$ \\
\hline LMC disk ......... & 0.99 & 0.3 & 1.05 & 1.06 & 50 & 36 & 50 \\
LMC halo........ & 0.94 & 0.1 & 1.44 & 1.53 & 100 & 25 & 106 \\
Galactic halo...... & 0.20 & 0.1 & 2.40 & 12.0 & 220 & 19 & 1100 \\
\hline
\end{tabular}

Note.-Also presented are the typical parameters for the geometry, $\left\langle D_{\mathrm{ol}} / D_{\mathrm{os}}\right\rangle$, the lens mass $\left\langle M_{L}\right\rangle$, and the transverse speed, $\langle v\rangle$, which are used in determining the expected $\left\langle\hat{r}_{\mathrm{E}}\right\rangle$ and $\langle v\rangle$. 
will be more useful than $\hat{r}_{\mathrm{E}}$ because $\hat{v}$ does not depend on the lens mass, which, for any given event, is completely unknown.

However, we note that for observations toward the Galactic bulge it is difficult to distinguish Galactic disk from Galactic bulge lenses using $\mu$ since both have similar distributions (Han \& Gould 1995).

\section{AN IMMEDIATE APPLICATION}

Although the events detected to date toward the LMC bear no obvious signatures of binary sources, it would nonetheless be interesting to check the sources for binarity. If any of the sources are binaries that would produce effects for the case of an LMC self-lensing event, the failure to detect an effect would prove that the lens was Galactic. To illustrate the nature of the required observations, suppose that one of the sources were an A-type binary with $M=5$ $M_{\odot}, \mathscr{Q}_{M}=2, \mathscr{Q}_{L}=16, P=100$ days, and a circular orbit. The velocity oscillations of the primary would then be sinusoidal with a full width $\Delta v=50 \mathrm{~km} \mathrm{~s}^{-1} \sin i$. Hence, for all but the rare nearly face-on orbits, the velocity variations would show up in three or four 1-2 $\mathrm{hr}$ observations on a $4 \mathrm{~m}$ telescope each separated $\sim 1$ month. Note that the velocity can be measured with an uncertainty of $\lesssim 10 \mathrm{~km}$ $\mathrm{s}^{-1}$ for 19 mag A-type and K giant stars (C. Pryor 1996, private communication). Other parameters considered in this paper $\left(P<100\right.$ days, $\left.\mathscr{Q}_{M}<2\right)$ would produce even stronger velocity variations. Once this source was identified as a binary candidate, it could be subjected to more intensive observation which would reveal the spectral class (and hence mass $M_{1}=3.3 M_{\odot}$ ) of the primary as well as the circular character of the orbit. Unless the observations were very intensive, the spectral signature of the secondary $(F$ star) might not be detected (single-line binary). For definiteness we assume that this lack of detection can be interpreted as an upper limit on its mass, $M_{2}<2 M_{\odot}$. Suppose for example $i=45^{\circ}$, so that $\Delta v=35 \mathrm{~km} \mathrm{~s}^{-1}$. Without detection of the secondary, this inclination would be unknown, but could be constrained to be $0.63<\sin i<1.0$ based on the mass ratio limit $\mathscr{Q}_{M}>1.65$. Hence the semimajor axis of the orbit of the primary would lie in the range $0.5 \mathrm{AU}<a_{1}<0.8 \mathrm{AU}$. Since this entire range is of the same order as $\hat{r}_{\mathrm{E}}$ for a typical LMC self-lensing event, there would be a dramatic effect on the light curve if the event were in fact of this type. Thus, in this case, one would determine that the event was Galactic even without detection of the secondary. This is in fact the case for most of the parameter space of short-period binary-source events.

In brief, the observations should follow three stages. First, sparse monitoring to determine which sources are binaries. Second, more intensive monitoring to determine the orbital elements and so possibly distinguish between Galactic and LMC events. Third, highly intensive follow up to try to detect the secondary if the questions about the event warrant this effort.

We would like to thank G. Newsom and C. Pryor for making useful comments and the referee for helpful suggestions. This work was supported by a grant AST 94-20746 from the NSF.

\section{REFERENCES}

Alard, C., Mao, S., \& Guibert, J. 1995, A\&A, 300, 17

Albrow, M., et al. 1996, in Proc. IAU Symp. 173, Astrophysical Applications of Gravitational Lensing, ed. C. S. Kochanek \& J. N. Hewitt (Dordrecht: Kluwer), 227

Alcock, C., et al. 1995, ApJ, 441, 21 1996a, ApJ, 461, 84

1996b, ApJ, 463, L67

1997a, ApJ, 479, 119

-1997b, ApJ, in press

Ansari, R., et al. 1995, A\&A, 299, L21

Axelrod, T., et al. 1994, BAAS, 184, 3302

Buchalter, A., \& Kamionkowski, M. 1997, ApJ, in press

Buchalter, A., Kamionkowski, M., \& Rich, R. M. 1996, ApJ, ApJ, 469, 676

Cook, K. H., et al. 1995, in Proc. IAU Symp. 155, Astrophysical Applica-

tions of Stellar Pulsations, ed. R. Stobie (Dordrecht: Kluwer), 221

Gould, A. 1992, ApJ, 392, 442 1994, ApJ, 421, L71

Gould, A., \& Welch, D. 1996, ApJ, 464, 212

Griest, K., \& Hu, W. 1992, ApJ, 397, 362
Grison, P., et al. 1995, A\&AS, 109, 447

Han, C., \& Gould, A. 1995, ApJ, 447, 53

Kałużny, J., et al. 1995, in ASP Conf. Ser., Binary Stars in Clusters, ed. G. Milone \& J. C. Mermilliod

Kamionkowski, M. 1995, ApJ, 442, L9

Loeb, A., \& Sasselov, D. 1995, ApJ, 449, L33

Mao, S., et al. 1994, BAAS, 185, 1705

Mao, S., \& Di Stefano, R. 1995, ApJ, 440, 22

Nemiroff, R. J., \& Wickramasinghe, W. A. D. T. 1994, ApJ, 424, L21

Pratt, M. R., et al. 1996a, in Proc. IAU Symp. 173, Astrophysical Applications of Gravitational Lensing, ed. C. S. Kochanek \& J. N. Hewitt (Dordrecht: Kluwer), 221

. 1996b, in Proc.: Sources and Dark Matter in the Universe, in press Sahu, K. 1994, Nature, 370, 275

Smart, W. 1962, Text-Book on Spherical Astronomy (Brook Crutchley: Cambridge Univ. Press), 360

Udalski, A., et al. 1994, ApJ, 436, 103

Witt, H. J. 1995, ApJ, 449, 42

Witt, H. J., \& Mao, S. 1994, ApJ, 430, 505 